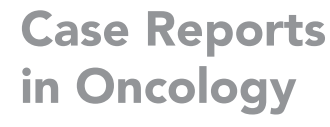

\title{
Successful Treatment with Nivolumab in a Patient with Metastatic Salivary Duct Carcinoma
}

\author{
Tsuyoshi Sato ${ }^{a}$ Takahiro Maeta $^{a}$ Ryosuke Abe ${ }^{b, c}$ Hiroyuki Yamada ${ }^{b}$ \\ Kazuyuki Ishidad $^{d}$ Akiko Yashima-Abo $^{\text {e }}$ Kazuki Kiyohara $^{a}$ \\ Shinri Miyajima ${ }^{a}$ Shigeki Ito $^{a}$ \\ aDivision of Hematology \& Oncology, Department of Internal Medicine, School of Medicine,

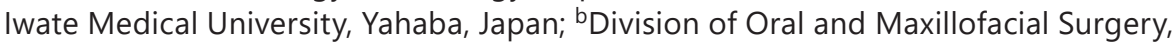 \\ Department of Reconstructive Oral and Maxillofacial Surgery, School of Dentistry, Iwate \\ Medical University, Yahaba, Japan; 'Department of Oral and Maxillofacial Surgery, Iwate \\ Prefectural Central Hospital, Morioka, Japan; ${ }^{\mathrm{d} D e p a r t m e n t ~ o f ~ D i a g n o s t i c ~ P a t h o l o g y, ~ D o k k y o ~}$ \\ Medical University, Mibu, Japan; ' Department of Pathology, School of Medicine, Iwate \\ Medical University, Yahaba, Japan
}

\section{Keywords}

Salivary duct carcinoma $\cdot$ PD-1 $\cdot$ Nivolumab

\section{Abstract}

Salivary duct carcinoma (SDC) is a rare and highly aggressive malignancy. A 58-year-old man presented with a right-sided submandibular mass and metastatic lesions in the right supraclavicular and inferior internal jugular nodes. He underwent right submandibulectomy and right neck dissection followed by adjuvant chemoradiotherapy. However, relapse occurred in the hilar lymph node and lumbar spine. Although radiotherapy was performed, a second relapse appeared in the hilar lymph nodes and sacral bone. Immunohistochemical analysis revealed negativity for programmed death ligand-1 (PD-L1) in the primary tumor specimen. The patient then received the anti-programmed death-1 (PD-1) antibody nivolumab. His metastatic lesions were completely eliminated after 48 weeks of therapy. This case reveals that anti-PD-1 antibodies are effective even against PD-L1-negative SDC. 


\section{Case Reports in Oncology}

\begin{tabular}{l|l}
\hline Case Rep Oncol 2021;14:343-346 \\
\hline DOI: 10.1159/000512060 & $\begin{array}{l}\text { ○ 2021 The Author(s). Published by S. Karger AG, Basel } \\
\text { www.karger.com/cro }\end{array}$ \\
\hline
\end{tabular}

Sato et al.: Nivolumab Therapy for Salivary Duct Carcinoma

\section{Introduction}

Salivary duct carcinoma (SDC) is a rare and highly aggressive malignancy [1]. Its prognosis is dismal, as more than $60 \%$ of patients die of relapse within 3 years [2, 3]. Therefore, novel treatments are necessary to improve outcomes. Nivolumab is a humanized monoclonal antibody targeting programmed death-1 (PD-1) that has displayed activity against recurrent squamous cell carcinoma of the head and neck [4]. However, its activity against rare subtypes of head and neck carcinoma such as SDC has not been elucidated. In this study, we have described the clinical course of a patient with metastatic SDC who was successfully treated with nivolumab.

\section{Case Presentation}

A 58-year-old man presented in July 2016 with a right-sided submandibular mass and metastatic lesions in the right supraclavicular and inferior internal jugular nodes. He underwent right submandibulectomy and right neck dissection in August 2016, which revealed SDC of the submandibular gland. Immunohistochemical observation illustrated that the tumor cells were positive for gross cystic disease fluid protein-15, androgen receptor, and human epidermal growth factor receptor 2 (2+ staining, shown in Fig. 1A-C). The patient then received adjuvant chemoradiotherapy with cisplatin. In April 2017, follow-up positron emission tomography/computed tomography (PET/CT) revealed metastatic lesions in the right hilar lymph node and lumbar spine. He underwent radiotherapy for both lesions. In January 2019, CT uncovered right hilar and upper mediastinal lymph node swelling and sacral bone metastasis (shown in Fig. 2A). The screening tests before nivolumab therapy revealed elevation of serum Krebs von den Lungen-6 (KL-6) levels $(1,007 \mathrm{U} / \mathrm{mL})$, although

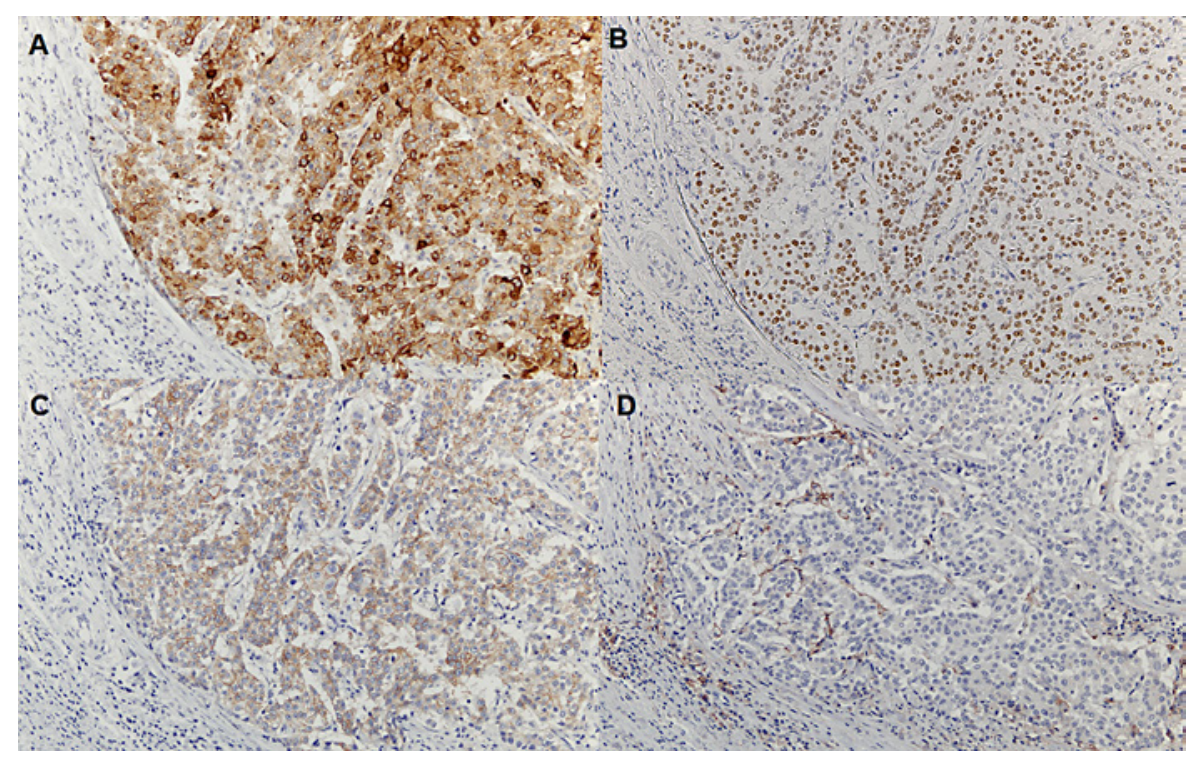

Fig. 1. Immunohistochemical findings of the salivary gland. A Gross cystic disease fluid protein-15-positive tumor cells are presented $(\times 200)$. B Androgen receptor-positive tumor cells are shown $(\times 200)$. C Human epidermal growth factor receptor 2 -positive $(2+)$ tumor cells are shown $(\times 200)$. D Programmed death ligand-1-negative tumor cells are presented $(\times 200)$. 


\section{Case Reports in Oncology}
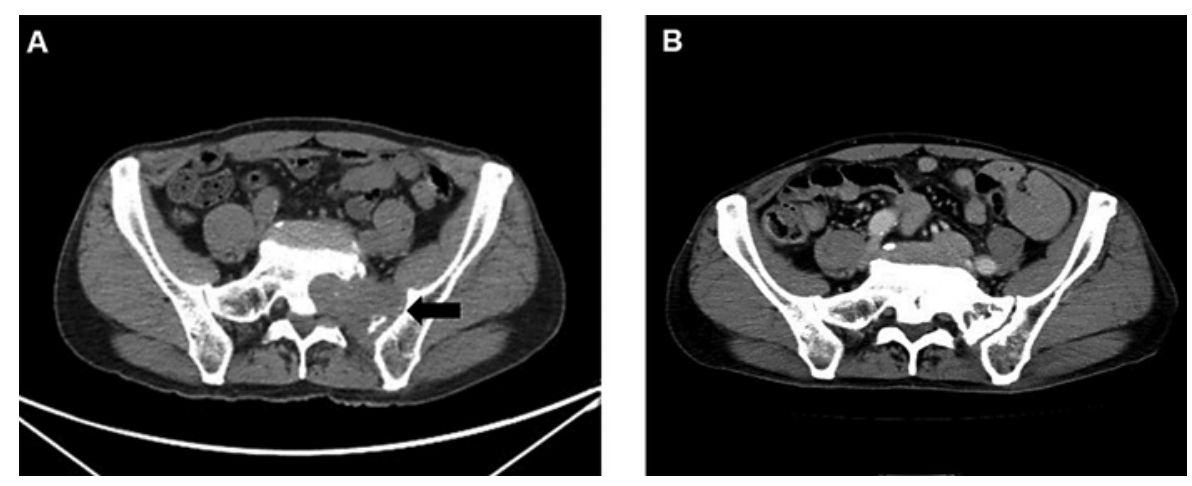

Fig. 2. Pelvic computed tomography findings. A Before nivolumab therapy. The arrow denotes the metastatic tumor of sacral bone. B After 48 weeks of nivolumab therapy. The sacral bone lesion was completely resolved.

CT demonstrated no evidence of interstitial pneumonia. Immunohistochemical analysis identified negativity for programmed death ligand-1 (PD-L1) and microsatellite instability in the primary tumor specimen (shown in Fig. 1D). In March 2019, intravenous nivolumab therapy was initiated at a dose of 240 mg every 2 weeks. Serum KL-6 levels normalized after 8 weeks of therapy, and CT scan showed interval shrinkage of the lesions in the hilar and mediastinal lymph nodes and sacral bone after 24 weeks of therapy. These metastatic lesions were completely resolved after 48 weeks of therapy (shown in Fig. 2B). The patient tolerated nivolumab with no adverse event.

\section{Discussion}

We have presented a case of the successful use of nivolumab in a patient with metastatic SDC even though the primary SDC specimen was negative for PD-L1 and microsatellite instability. A recent study demonstrated that PD-L1 was expressed in more than $50 \%$ of patients with SDC specimens [5]. The anti-PD-1 antibody pembrolizumab was linked to response and stable disease rates of 12 and 26\%, respectively, in a small expansion cohort of 26 patients with advanced salivary gland cancer including one patient with SDC who did not achieve a partial response [6]. Harwood et al. [7] recently reported a case of durable remission in a patient with metastatic PD-L1-positive SDC who was treated with pembrolizumab. These results suggest that anti-PD-1/anti-PD-L1 may have some antitumor activity against this disease. In our case, nivolumab exhibited antitumor activity even though the primary tumor was negative for PD-L1. Because radiotherapy was applied to the bone and lymph node metastatic regions before nivolumab therapy, the efficacy of the drug might have been enhanced by radiotherapy-induced abscopal effects.

Interestingly, serum KL-6 levels were elevated before treatment in our case. The KL-6 antigen was initially recognized as a tumor marker [8]. The serum KL-6 level is also a diagnostic marker of interstitial pneumonia. In our case, CT revealed no evidence of interstitial pneumonia despite elevation of the serum KL-6 level, and the level normalized with tumor shrinkage during nivolumab therapy, suggesting that serum KL- 6 was a tumor marker in this case. To our knowledge, this is the first reported case of successful treatment with nivolumab in a patient with metastatic PD-L1-negative SDC. Treatment with immune checkpoint inhibitors should be further investigated even in patients with PD-L1-negative SDC.

\section{Karger'}




\section{Case Reports in Oncology}

Case Rep Oncol 2021;14:343-346

\begin{tabular}{l|c|c|}
\hline DOI: $10.1159 / 000512060$ & $\odot 2021$ The Author(s). Published by S. Karger AG, Basel
\end{tabular} www.karger.com/cro

\section{Statement of Ethics}

Written informed consent was obtained from the patient for publication of this case report and any accompanying images.

\section{Conflict of Interest Statement}

S.I. received honorarium from Ono and Bristol-Myers Squibb (BMS).

\section{Funding Sources}

No funding was received for this article and the authors have no conflicts of interest directly relevant to this report.

\section{Author Contributions}

Conception and design: Tsuyoshi Sato, Takahiro Maeta, Shigeki Ito. Collection and assembly of data: Tsuyoshi Sato, Kazuyuki Ishida, Akiko Yashima-Abo, Shigeki Ito. Data analysis and interpretation: Tsuyoshi Sato, Kazuyuki Ishida, Akiko Yashima-Abo, Shigeki Ito. Manuscript writing: all authors. Final approval of manuscript: all authors. Accountable for all aspects of the work: all authors.

\section{References}

1 Speight PM, Barrett AW. Salivary gland tumours. Oral Dis. 2002;8(5):229-40.

2 Jayaprakash V, Merzianu M, Warren GW, Arshad H, Hicks WL, Rigual NR, et al. Survival rates and prognostic factors for infiltrating salivary duct carcinoma: analysis of 228 cases from the Surveillance, Epidemiology, and End Results database. Head Neck. 2014;36(5):694-701.

3 Gilbert MR, Sharma A, Schmitt NC, Johnson JT, Ferris RL, Duvvuri U, et al. A 20-year review of 75 cases of salivary duct carcinoma. JAMA Otolaryngol Head Neck Surg. 2016;142(5):489-95.

4 Ferris RL, Blumenschein G Jr, Fayette J, Guigay J, Colevas AD, Licitra L, et al. Nivolumab for recurrent squamouscell carcinoma of the head and neck. N Engl J Med. 2016;375(19):1856-67.

5 Mukaigawa T, Hayashi R, Hashimoto K, Ugumori T, Hato N, Fujii S. Programmed death ligand-1 expression is associated with poor disease free survival in salivary gland carcinomas. J Surg Oncol. 2016;114(1):36-43.

6 Cohen RB, Delord JP, Doi T, et al. Pembrolizumab for the treatment of advanced salivary gland carcinoma: findings of the phase 1b KEYNOTE-028 Study. Am J Clin Oncol. 2018;41:1083-8.

7 Harwood TC, Oliver AS, King S, Shahlapour M, Dhillon WS. Long-term durable response after treatment of metastatic salivary duct carcinoma with pembrolizumab. JCO Oncol Pract. 2020;16(7):397-8.

8 Kohno N, Inoue Y, Hamada H, Fujioka S, Fujino S, Yokoyama A, et al. Difference in sero-diagnostic values among KL-6-associated mucins classified as cluster 9. Int J Cancer Suppl. 1994;8:81-3. 